\title{
Testing the shifting defense hypothesis for constitutive and induced resistance and tolerance
}

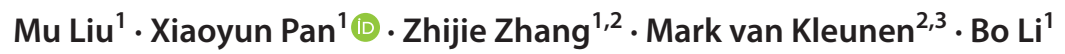

\begin{abstract}
Biogeographical variation in herbivore communities may drive invasive plants to evolve lower defense against specialist herbivores but higher defense against generalist herbivores (shifting defense hypothesis, SDH). However, empirical tests on this topic have been strongly biased toward examining constitutive resistance and less is known about the evolution of induced resistance and tolerance. We examined constitutive and induced resistance and tolerance of the invasive plant, Alternanthera philoxeroides (alligator weed, Centrospermae: Amaranthaceae) against the specialist herbivore Agasicles hygrophila (Coleoptera, Chrysomelidae) and the generalist herbivore Spodoptera litura (Lepidoptera, Noctuidae), using genotypes from its introduced and native ranges. We found that introduced genotypes, compared to native genotypes, had higher constitutive resistance to the generalist herbivore, but similar constitutive resistance to the specialist herbivore. Furthermore, introduced genotypes, compared to native genotypes, had lower induced resistance to the generalist herbivore, but similar induced resistance to the specialist herbivore. Moreover, although introduced and native genotypes did not differ in their tolerance to the generalist, the introduced genotypes exhibited lower tolerance to the specialist than native ones. Therefore, while individual defense strategy does not indicate a shift from defense against the specialist to defense against the generalist, the results for the different defense strategies jointly support the SDH.
\end{abstract}

Keywords Biological invasion · Biological control $\cdot$ Enemy release hypothesis $\cdot$ Phenotypic plasticity $\cdot$ Plant-herbivore interactions · Trade-offs

\section{Key message}

Xiaoyun Pan

xypan@fudan.edu.cn

Mu Liu

lium16@fudan.edu.cn

Zhijie Zhang

zhijie.zhang@uni-konstanz.de

Mark van Kleunen

mark.vankleunen@uni-konstanz.de

Bo Li

bool@fudan.edu.cn
- Experimental studies have seldom considered multiple defensive strategies simultaneously to assess the possible evolution of defense in invasive plants against both specialist and generalist herbivores.

- Introduced genotypes exhibited decreased induced resistance against generalist, lower tolerance to specialist her-

Ministry of Education Key Laboratory for Biodiversity Science and Ecological Engineering, Coastal Ecosystems Research Station of the Yangtze River Estuary, Institute of Biodiversity Science and Institute of Eco-Chongming, School of Life Science, Fudan University, Shanghai, China

2 Ecology, Department of Biology, University of Konstanz, Constance, Germany

3 Zhejiang Provincial Key Laboratory of Plant Evolutionary Ecology and Conservation, Taizhou University, Taizhou, China 
bivores, and greater constitutive resistance against generalist herbivores compared to native genotypes.

- It is critical to focus on all three defensive strategies when we examine the evolution of plant defense to develop efective methods for biological control of invasive plants.

\section{Introduction}

Invasive plants may be released from specialist herbivores and simultaneously face biotic resistance from resident generalist herbivores in their introduced ranges (Keane and Crawley 2002). The shifting defense hypothesis (SDH) predicts that such shifts in the composition of herbivore communities will result in the evolution of decreased defense against specialist herbivores and increased defense against generalist herbivores (Joshi and Vrieling 2005; MüllerSchärer et al. 2004).

Plant defense against herbivores consists of two major strategies-resistance and tolerance. Resistance reduces a herbivore's preference for the plant or the performance of the herbivores (Strauss et al. 2002), whereas tolerance mitigates negative impacts of damage on plant fitness (Strauss and Agrawal 1999). Resistance can be further classified into constitutive and induced resistance. Constitutive resistance is constantly maintained regardless of the presence of herbivores, whereas induced resistance is triggered by herbivore attack (Karban and Baldwin 1997).

Despite accumulating evidence for biogeographical variation in constitutive resistance against specialists and generalists (reviewed in Zhang et al. 2018), induced resistance and tolerance have been rarely considered in studies testing the SDH (Table 1). We found 11 previous studies that tested the evolution of defense against both specialist and generalist herbivores in invasive plants (Table 1) and measured resistance in terms of herbivore performance. Four of the 11 previous studies provide evidence supporting the SDH, as they found that introduced genotypes, compared to native genotypes, had lower constitutive resistance or tolerance to specialists but higher constitutive resistance or tolerance to generalists. Three studies provide partial evidence supporting the SDH, as they found that either resistance to specialists or resistance to generalists changed in the direction predicted by the SDH. The results of the remaining four studies are inconsistent with the predictions of the SDH. Interestingly, these latter studies primarily focused on a single defensive strategy, in most cases constitutive resistance (the last two categories in Table 1). This indicates that it might be essential to consider multiple defense strategies (i.e., constitutive and induced resistance and tolerance) when testing the SDH.

We used Alternathera philoxeroides (alligator weed, Centrospermae: Amaranthaceae) to examine biogeographical variation in plant defense strategies against the specialist Agasicles hygrophila (Coleoptera: Chrysomelidea) and the generalist Spodoptera litura (Lepidoptera: Noctuidae). This herbaceous plant species is native to South America and has become invasive in parts of USA, Australia, and China (Julien et al. 1995). In a previous study, we found that introduced and native genotypes of $A$. philoxeroides had comparable constitutive resistance against the specialist herbivore A. hygrophila (Pan et al. 2012). However, we also found that introduced and native genotypes differed in induced defenses against specialist and generalist herbivores (Liu et al. 2018). For example, introduced genotypes responded with lower concentrations of total triterpenoid saponins when damaged by the generalist Spodoptera litura than when damaged by the specialist A. hygrophila (Liu et al. 2018). However, for native genotypes, the increase in the concentrations of total triterpenoid saponins did not depend on whether the damage was caused by the specialist or generalist (Liu et al. 2018). Building on those previous studies, we asked whether the variation of constitutive resistance, induced resistance, and tolerance to specialist and generalist herbivores between native and introduced genotypes of $A$. philoxeroides is consistent with the predictions of SDH.

\section{Materials and methods}

\section{Study species}

We focus in this study on the interaction between a worldwide invasive plant Alternanthera philoxeroides and two phytophagous insect species, the specialist Agasicles hygrophila and the generalist Spodoptera litura. Alternanrhera philoxeroides is native to South America, spanning a wide distribution range from 18 to $39^{\circ} \mathrm{S}$. It has been globally introduced to regions from warm to subtropical humid climates (Pan et al. 2007). Alternanthera philoxeroides is known to be fed on by at least 40 herbivore species in its home range, including at least five specialists (Sosa et al. 2004). In its introduced range in USA, there are few resident herbivores feeding on A. philoxeroides (Maddox et al. 1971).

Agasicles hygrophila and its congeners are host-specific to A. philoxeroides, and in South America both adults and larvae of these herbivores feed on leaf and stem tissue of A. philoxeroides in terrestrial and aquatic habitats (Cabrera et al. 2013; Sosa et al. 2004). Agasicles hygrophila was introduced as a biological control agent in USA since the 1960s and is the primary specialist herbivore in this region (Pan et al. 2007). However, in the introduced range, it is still restricted to a limited number of habitats and has a preference for aquatic habitats (Maddox et al. 1971; Pan et al. 2007). Therefore, enemy release may continue to act 
Table 1 Summary of results of 11 previous studies (1-4 and 6-12) and the current study (5) testing for plant defense strategies of invasive plants against specialist and generalist herbivores. + (yellow triangle): higher defense in introduced than native genotypes; - (blue triangle): lower defense in introduced than native genotypes; 0 (gray triangle): similar defense for the two plant origins. Studies marked on the left side in green: results for plant defenses against specialist and generalist herbivores both support the SDH. Studies marked on the left side in yellow: only the result for plant defense against specialist or the result for plant defense against generalist herbivores support the SDH. Studies marked on the left side in red: results for plant defenses against specialist and generalist herbivores both do not support the SDH

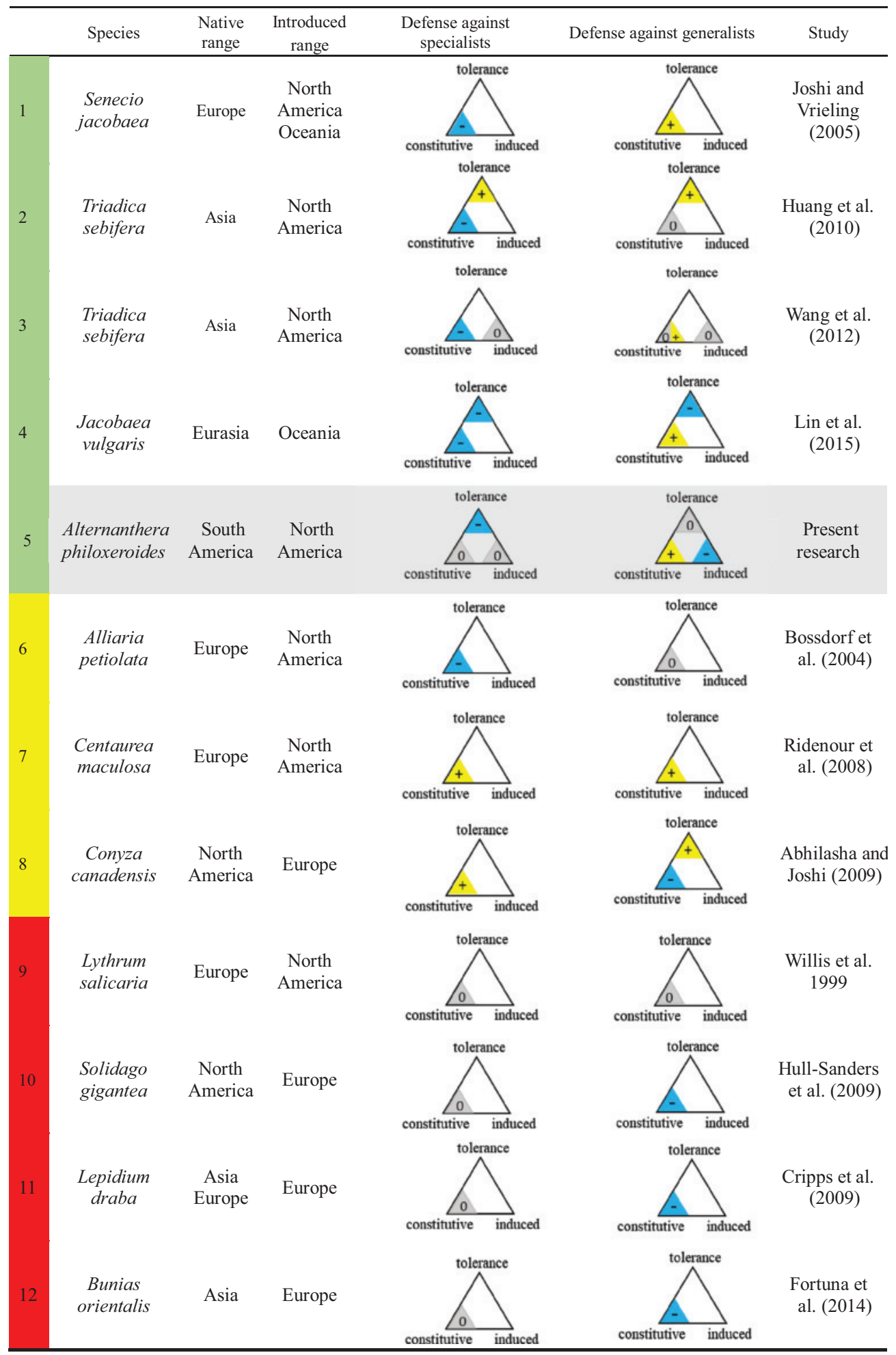


on the introduced $A$. philoxeroides populations growing in terrestrial habitats of USA (van Klinken and Edwards 2002).

Spodoptera litura (Lepidoptera: Noctuidae) and its congeners are generalist defoliators (Rao et al. 1993), which can feed on leaves of A. philoxeroides. The genus has a wide global geographical range (Saldamando and Marquez 2012). In North America, these herbivores are restricted to southern Florida and Texas during winter, but infestations extend as far north as Canada during summer and fall (Luginbill 1928).

\section{Source populations of plants and herbivores}

For the present study, we obtained stem fragments from five native (Argentina) and five introduced (USA) source plants from distinct geographical regions in 2005 and 2006, respectively. We sampled A. philoxeroides plants in terrestrial habitats only to ensure that the USA plants had not been exposed to the specialist $A$. hygrophila. Detailed information on the sample sites is provided in Liu et al. (2018). One of our previous studies showed that each sampled plant was characterized by a unique multi-locus genotype (Geng et al. 2016). Plants were propagated in a glasshouse for at least three generations to avoid possible maternal carryover effects (Schwaegerle et al. 2000; Dong et al. 2018).

We collected larvae of $A$. hygrophila in fields in Shanghai, China, where it has been introduced as a biological control agent. These introduced beetle populations originated from the Ezeiza Lagoon area near Buenos Aires, Argentina, and were introduced into China in the 1980s (Li and Wang 1994). We raised the beetles on potted A. philoxeroides in Shanghai, and their offspring were used for the experiment. Larvae of the generalist $S$. litura were purchased from a company (Ke Yun biological control Co., Ltd., Henan, China) in Henan province, China, and were raised on an artificial diet before use in the experiment (main nutrients of artificial diet in Cao et al. 2014).

\section{Common garden experiments}

\section{Propagation}

On August 21, 2015, we placed stem fragments (4 cm long, with one node) in 150-mm Petri dishes lined with moist filter paper at $25 / 28{ }^{\circ} \mathrm{C}(12 / 12 \mathrm{~h})$ to promote root and shoot development. On August 28, 2015, we transplanted the plants to pots $(7 \mathrm{~cm}$ diameter and $12 \mathrm{~cm}$ high) filled with c. 400-ml commercial potting compost (Beilei Organic Fertilizer Co., Ltd., Zhenjiang, China).

\section{Constitutive and induced resistance}

On September 21, 2015, we conducted two experiments to measure constitutive and induced resistance against a specialist and a generalist herbivore. Firstly, we selected 80 plants ( 8 for of each genotype) of similar size (i.e., a height of $c .10 \mathrm{~cm}$, with four pairs of leaves) and randomly assigned them to two treatments: (1) herbivory by the specialist $A$. hygrophila or (2) control (without herbivory). Secondly, we selected another 80 plants ( 8 for of each genotype) of similar size and randomly assigned them to either (1) herbivory by the generalist $S$. litura or (2) control (without herbivory). Each genotype-by-treatment combination in both experiments were replicated four times. For the A. hygrophila and $S$. litura herbivory treatments, we exposed the plants to herbivory by two larvae of the respective herbivores for 4 days. On each plant, these two larvae were confined to two fully expanded young leaves by enclosing them separately into two centrifuge tubes. This ensured that they removed c. $25 \%$ of the total leaf area (Appendix S1). In the control treatment, we also had two centrifuge tubes on each plant, but these did not receive any larvae. After 4 days, we removed all herbivores and tubes.

On October 1, 2015, to measure constitutive resistance to the specialist $A$. hygrophila, plants grown in the control treatment were fed to three larvae of specialist A. hygrophila. Specifically, we chose similar larvae of the same instar to control for variation in initial weight of the larvae. After pre-weighing the three larvae, we let them eat the individual plant freely in the bag and collected them from the bag after 3 days and weighed them jointly again to track the total mass gain of all three larvae. We determined the combined mass of the three larvae before and after feeding on the plants to the nearest $0.001 \mathrm{mg}$. To measure induced resistance to the specialist $A$. hygrophila, plants previously exposed to A. hygrophila herbivory were fed to A. hygrophila again, as described above for the measurement of constitutive resistance. To measure constitutive and induced resistance to the generalist $S$. litura, we confined three larvae of $S$. litura in a nylon mesh bag to each plant for 3 days. We determined the combined mass of the three larvae before and after feeding on the plants to the nearest $0.001 \mathrm{mg}$. For these bioassays, we used second-instar larvae of A. hygrophila and thirdinstar larvae of $S$. litura, because a pilot experiment had shown that these two instar classes of larvae have nearly equivalent feeding rates (unpublished data).

Constitutive resistance was quantified in terms of the total mass gain of three A. hygrophila or S. litura larvae fed on the control plants, where a low mass gain indicates a high constitutive resistance. Induced resistance was quantified by comparing the mass gain of herbivores fed on the control plants and the mass gain of those fed on the herbivory treatment plants. Here, induced resistance was indicated by 
a lower herbivore mass gain on plants from the herbivory treatment than those from the control treatment.

To test whether introduced and native genotypes of $A$. philoxeroides differed in their constitutive resistance, total mass gain of the specialist $A$. hygrophila and the generalist $S$. litura raised on control plants were analyzed using GLMMs with a Gaussian error distribution and a log-link function (which effectively log transforms the response variable to improve normality and homoscedasticity of the residuals). We assigned plant origin (introduced vs. native), herbivore type (specialist vs. generalist), and their interaction as fixed terms, and genotype as a random effect.

To test whether introduced and native genotypes of $A$. philoxeroides differed in their induced resistance, we analyzed the bioassay dataset that included the specialist $A$. hygrophila raised on plants from both the control treatment and the A. hygrophila herbivory treatment, and the bioassay dataset that included the generalist $S$. litura raised on plants from both the control treatment and the S. litura herbivory treatment. Mass gain of specialist and generalist herbivores was analyzed using GLMMs with a Gaussian error distribution and with a log-link function. We assigned plant origin (introduced vs. native), herbivory treatment (herbivore present vs. control) and their interaction as fixed terms, and genotype as a random effect.

\section{Tolerance and plant defense trait measurements}

In parallel to the constitutive and induced resistance assays, we conducted another experiment to measure tolerance and plant traits related to defense. We selected 120 plants (12 of each genotype) of similar size (i.e., a height of $c .10 \mathrm{~cm}$, with four pair of leaves) and randomly assigned these plants to three treatments: (1) herbivory by the specialist $A$. hygrophila (2) herbivory by the generalist S. litura, or (3) control (without herbivory). Each genotype-by-treatment combination was replicated four times. Plants in those treatments were handled in the same manner as described above for the plants in the constitutive and induced resistance assays, but they did not receive any bioassay larvae. We harvested the plants 14 days after removal of the herbivory treatments. Considering that we conducted the study with plants that were in the early stages for their life cycle, the 14 days of recovery should have been enough to exhibit effects of herbivory on plant fitness (Quintero and Bowers 2013).

To quantify the tolerance to herbivory, we measured the aboveground, belowground and total biomass of each plant, which is likely to be closely related to fitness of $A$. philoxeroides, as regeneration of the species mainly depends on vegetative propagation (Sosa et al. 2004). We cut the aboveground biomass and washed the belowground biomass free of soil. The biomass was then dried to constant mass at $60{ }^{\circ} \mathrm{C}$ and weighed to the nearest $0.001 \mathrm{mg}$. When comparing plants from the herbivory treatments with those from the control treatment, a similar total plant biomass indicates a high tolerance to herbivory (Strauss and Agrawal 1999).

To measure concentrations of triterpenoid saponins, the major defense chemicals produced by A. philoxeroides (Faizal and Geelen 2013), leaves of four plants within the same genotype-by-treatment combination were pooled to get sufficient material, and ground. The total triterpenoid saponins concentrations was assessed by UV spectrophotometry (Wang et al. 2011). The leaf powder (c. $20 \mathrm{mg}$ ) was steeped in diethyl ether, heated in a water bath $\left(30^{\circ} \mathrm{C}\right.$ for $4 \mathrm{~h}$ ), and then centrifuged. We extracted the triterpenoid saponins from the residue successively with methyl alcohol and chloroform, and then we desiccated the chloroform. Finally, $200 \mu \mathrm{l}$ methyl alcohol was used to dissolve the extract and we measured absorbance of the solution at $215 \mathrm{~nm}$. Oleanolic acid was used as a standard for regression equations used to calculate the total triterpenoid saponins concentrations.

To test whether introduced and native genotypes of $A$. philoxeroides differed in their tolerance to herbivory, we analyzed the plant traits dataset that included plants grown in the control, A. hygrophila herbivory and S. litura herbivory treatments. Total plant biomass was analyzed using GLMMs with a Gaussian error distribution and a log-link function. We assigned plant origin (introduced vs. native), herbivory treatment (control, A. hygrophila herbivory and S. litura herbivory) and their interaction as fixed terms, and genotype as a random effect. Additionally, we also analyzed root-shoot ratio using the same model as for total plant biomass and found no significant effect of the herbivory treatment and no significant interaction between plant origin and herbivory treatment, indicating above and belowground biomass had similar responses to herbivory regardless of plant origin (Appendix S2). Therefore, we only present total plant biomass as indicator of plant performance.

To test whether introduced and native genotypes of $A$. philoxeroides differed in their concentrations of total triterpenoid saponins and the responses to different herbivory treatments, we analyzed the plant traits dataset. The concentrations of total triterpenoid saponins was analyzed using LMMs. We assigned plant origin (introduced vs. native), herbivory treatment (control, A. hygrophila herbivory and $S$. litura herbivory), and their interaction as fixed terms, and genotype as a random effect. Because leaves had to be pooled across the four plants within each genotype-by-treatment combination, we analyzed the genotype-level data for concentrations of total triterpenoid saponins.

To examine the correlation between the concentrations of total triterpenoid saponins and specialist or generalist herbivore performance, we used LMMs and assigned mass gain of the specialist A. hygrophila or the generalist S. litura 
larvae as the response variable and concentrations of total triterpenoid saponins, herbivory treatment (herbivore present vs. control), and their interaction as fixed terms, and genotype as the random effect. A significant effect of the total triterpenoid saponins term indicates that there is significant correlation between the concentrations of total triterpenoid saponins and herbivore performance.

We performed all analyses in R 3.5.2 (R Development Core Team 2017). We used the lmer package (Bates et al. 2015) for generalized linear mixed-effects models (GLMMs) and linear mixed-effects models (LMMs), and the agricolae package (De Mendiburu 2009) for least significant difference (LSD) post hoc tests. The significance of fixed terms in the mixed models was assessed with likelihood ratio tests when comparing models with and without the effect of interest (Zuur et al. 2009). When a significant interaction was detected, we performed LSD post hoc tests to contrast specific means and used different letters in the figures to indicate significant differences $(P<0.05)$.

\section{Results}

\section{Constitutive and induced resistance}

When raised on control plants, specialist and generalist herbivores had significantly different performance depending on plant origin (significant origin-by-herbivore type interaction, Fig. 1a), indicating differences in constitutive resistance of plants. The specialist $A$. hygrophila had a similar mass gain when raised on introduced or native plant genotypes (Fig. 1a). However, the generalist $S$. litura had a significantly
Fig. 1 Plant defense strategies against specialist and generalist herbivores. a Constitutive resistance: mass gain of specialist and generalist herbivores raised on native (Argentina; gray bars) and introduced (USA; open bars) genotypes of Alternanthera philoxeroides grown in the control treatment (i.e., without previous herbivory). Induced resistance: the effects of previous herbivory by $\mathbf{b}$ the specialist Agasicles hygrophila and $\mathbf{c}$ the generalist Spodoptera litura on mass gain of subsequent conspecific larvae raised on native (gray bars) and introduced (open bars) genotypes of A. philoxeroides. d Tolerance: the effects of herbivory by the specialist and generalist herbivores on total plant biomass of native (gray bars) and introduced (open bars) genotypes of $A$. philoxeroides. Values are means \pm SE. Significance of the a plant origin-by-herbivore types interaction and $\mathbf{b}, \mathbf{c}, \mathbf{d}$ the plant origin-by-herbivory treatments interaction are indicated. Different letters indicate significant differences at $P<0.05$ according to LSD post hoc contrasts
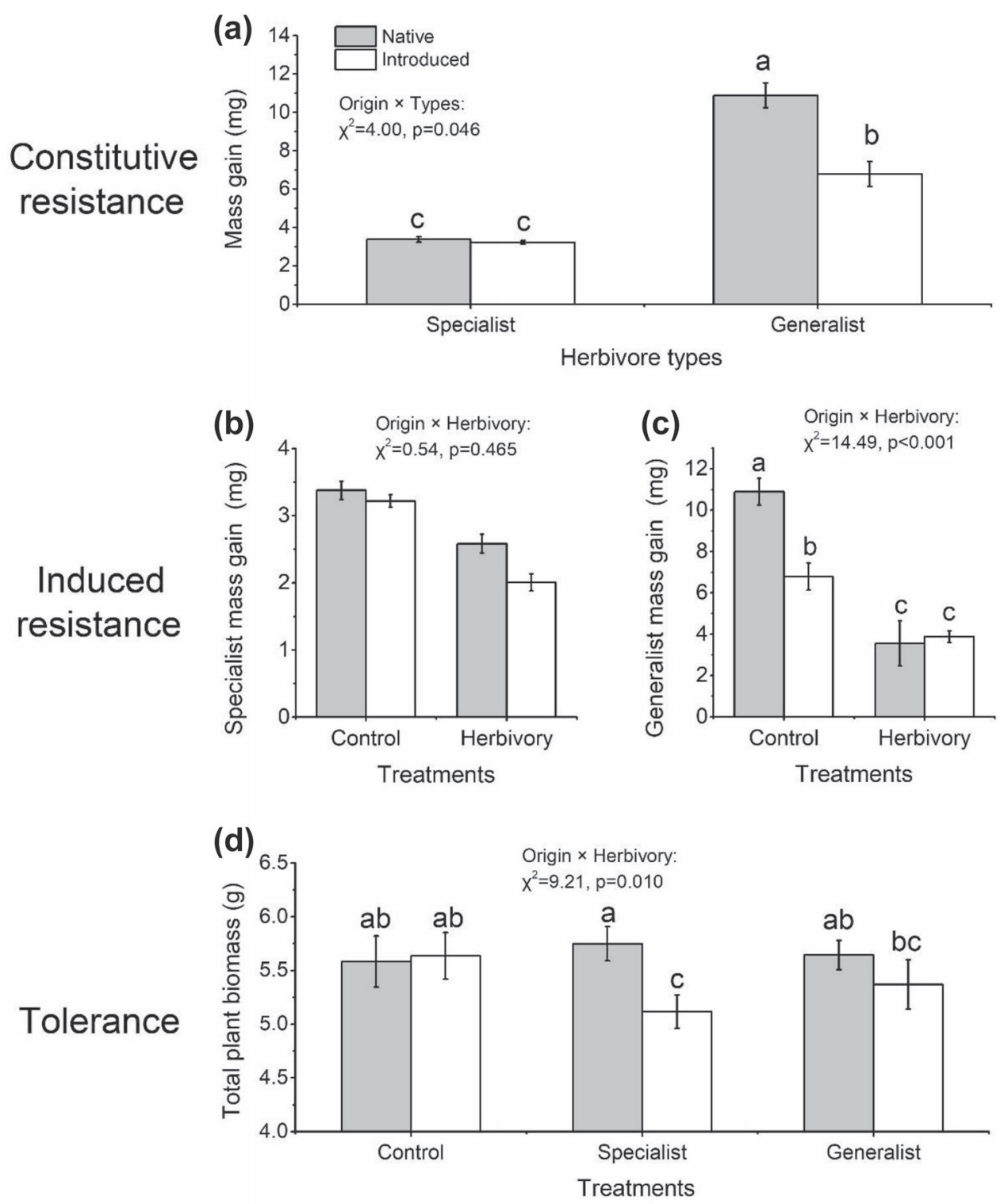
lower mass gain when raised on introduced plant genotypes than when raised on native genotypes (Fig. 1a).

Both the specialist $A$. hygrophila and the generalist $S$. litura raised on plants previously exposed to herbivores had significantly lower mass gain than those raised on control plants $\left(\chi^{2}=12.89, P<0.001\right.$ for specialist; $\chi^{2}=49.59$, $P<0.001$ for generalist), indicating induced resistance. For the specialist $A$. hygrophila, this negative effect of previous herbivory on subsequent herbivore performance did not depend on the origin of the plant hosts (Fig. 1b). However, for the generalist $S$. litura, the negative effect of previous herbivory was stronger when larvae were raised on native plant genotypes than when raised on introduced genotypes (Fig. 1c).

\section{Tolerance and plant defense trait measurements}

Introduced and native plant genotypes significantly differed in their responses of total biomass to different herbivory treatments (significant origin-by-herbivore type interaction, Fig. 1d), indicating differences in tolerance. Introduced plant genotypes fed upon by the specialist $A$. hygrophila had lower total biomass than plants without herbivory, whereas this was not the case for native plant genotypes (Fig. 1d). Biomass for native and introduced plant genotypes were similar regardless of herbivory by the generalist $S$. litura.

In the control treatment, introduced plant genotypes had a significantly higher concentrations of total triterpenoid saponins than native genotypes (Fig. 2a). Introduced plant genotypes had similar concentrations of total triterpenoid saponins regardless of herbivore treatment. Native plant genotypes had a significantly higher concentrations of total triterpenoid saponins compared with the control treatment (Fig. 2a). The concentrations of total triterpenoid saponins were negatively correlated with mass gain of the generalist $S$. litura, but it was not significantly correlated with mass gain of the specialist $A$. hygrophila (Fig. 2b, c).

\section{Discussion}

Our major finding is that introduced genotypes of Alternanthera philoxeroides, compared to native genotypes, showed higher constitutive resistance against the generalist Spodoptera litura and lower tolerance to the specialist Agasicles hygrophila (Fig. 1). This finding is consistent with the predictions of the shifting defense hypothesis (SDH, Table 1). However, while we found support for SDH across different defense strategies, we did not find consistent support for SDH within any single defense strategy. For example, although introduced genotypes had, relative to native genotypes, an enhanced constitutive resistance against the generalist herbivore, they did not show a significant decrease in constitutive resistance against the specialist herbivore (Fig. 1).Thus, our results indicate that the shift from defenses against specialists to generalists, as predicted by the SDH may involve different defense strategies against each of the two types of herbivores.

A possible explanation for our finding is that specialist and generalist herbivores may select for different defense strategies. Previous studies indicated that tolerance is likely to be selected for in a location dominated by specialist herbivores, while resistance may be favored in a location with more generalized herbivores (Carmona and (a)

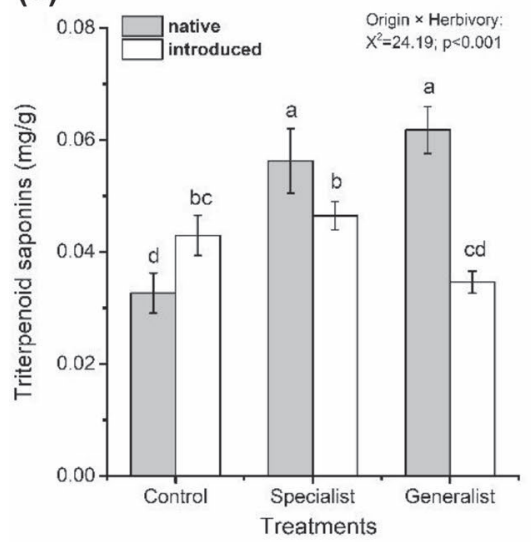

(b)

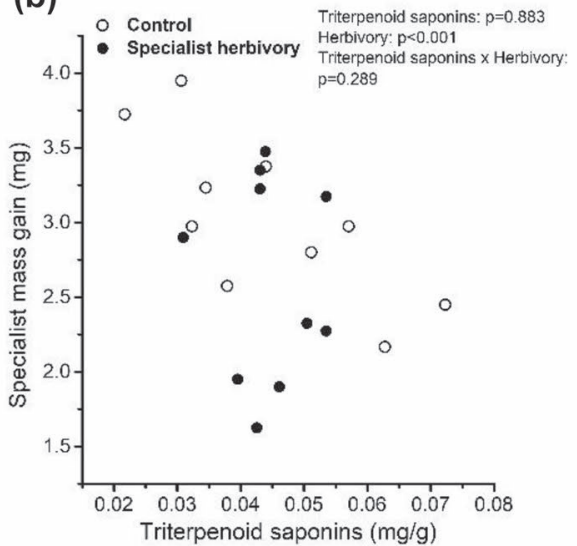

(c)

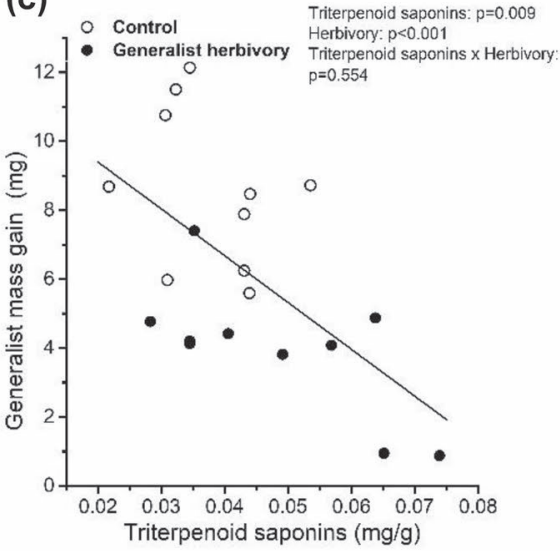

Fig. 2 a The effects of herbivory by specialist (Agasicles hygrophila) and generalist (Spodoptera litura) herbivores on total triterpenoid saponins of native (Argentina, gray bars) and introduced (USA, open bars) genotypes of Alternanthera philoxeroides, and $\mathbf{b}$, $\mathbf{c}$ the correlation between the concentrations of total triterpenoid saponins and mass gain of the two herbivores. Values in (a) are means \pm SE. Sig- nificance of the plant origin-by-herbivory treatment interaction is indicated. Different letters indicate significant differences at $P<0.05$ according to LSD post hoc contrasts. Values in $(\mathbf{b}, \mathbf{c})$ are genotypelevel data in both control (open circles) and herbivory treatment (solid circles). Significance of the fixed terms on herbivore mass gain is indicated 
Fornoni 2013; Fornoni et al. 2004). Furthermore, it has been suggested that tolerance might play a more important role in plant defense against specialist herbivores, because specialists will cause damage irrespective of plant resistance (Ali and Agrawal 2012), and in that way, they could cause more severe damage than generalist herbivores do (Lin et al. 2015). This would imply that, in the introduced range, the release from specialist herbivores and the encounter of new generalist herbivores would select for lower tolerance but higher resistance in plants. This prediction is supported by our study and some previous studies (Lin et al. 2015; Oduor et al. 2011). For example, introduced populations of Brassica nigra showed higher resistance but lower tolerance to herbivory than native populations (Oduor et al. 2011). However, these results are not consistent with results for Triadica sebifera, the only invasive woody species we found in our review (Table 1). Huang et al. (2010) found that introduced T. sebifera genotypes had higher tolerance than native genotypes to both specialist and generalist herbivory. The inconsistency with our findings could suggest that life form (woody vs. herbaceous) may affect the evolution of defense strategies (Haukioja and Koricheva 2000).

Another prediction of the SDH is that introduced plant genotypes could evolve to increase their cheap, qualitative defensive traits like toxins and reduce their expensive quantitative defensive traits like digestibility reducing compounds (Müller-Schärer et al. 2004). Although a growing number of recent studies have compared defensive traits between plants from the native and introduced ranges (Doorduin and Vrieling 2011), it is still unknown whether the evolution of defensive traits in invasive species could translate into the evolution of resistance in terms of performance of herbivores (Zhang et al. 2018). In our study, introduced genotypes showed a higher constitutive but lower induced level of total triterpenoid saponins than native genotypes, and there was a negative correlation between the concentrations of total triterpenoid saponins and performance of the generalist $S$. litura (Fig. 2c). Therefore, we speculate that triterpenoid saponins play the role of qualitative defensive trait of $A$. philoxeroides and that geographical variation of resistance to the generalist herbivore may derive from variation in constitutive and induced total triterpene saponins concentrations (Fig. 2).

Our results are highly relevant for biological control of A. philoxeroides. Although introduced and native genotypes of $A$. philoxeroides had similar constitutive and induced resistance against the specialist $A$. hygrophila, which has been introduced as a biological control agent, the decreased tolerance to specialist herbivory in introduced genotypes may contribute to the effectiveness of this biological control agent. Therefore, greater consideration of variation of different plant defensive strategies between introduced and native plant genotypes may lead to a higher success rate in developing effective methods for biological control agents of invasive plants (Zhang et al. 2018).

In conclusion, although the SDH has been tested in various study systems, most studies focused only on one or two plant defense strategies to specialist and generalist herbivores. In our study, introduced genotypes increased their constitutive resistance against a generalist herbivore at the cost of decreased induced resistance to this herbivore and tolerance to a specialist herbivore. Therefore, it is important to focus on all three defensive strategies simultaneously when we examine the evolution of plant defense to develop efective methods for biological control of invasive plants.

\section{Author contribution}

ML and XP designed the experiment; ML performed the experiment; ML and ZZ analyzed the data; ML wrote the paper, with major inputs from XP and MK, and further inputs from all other authors.

Acknowledgements The work was supported by the National Key Research and Development Program of China (2017YFC1200100) and National Natural Science Foundation of China (Grant No. 41771053). We thank Dana Blumenthal (USDA-ARS Rangeland Resources Research Unit, Fort Collins, CO) for the critical reading of the manuscript and the valuable comments. We also thank Alejandro Sosa (The Foundation for the Study of Invasive Species, Spanish acronym: FuEDEI) for providing clones of the Argentinian plant genotypes. We are also grateful to Xiang Liu, Youzheng Zhang, and other members of IBSFU for their assistance in conducting the glasshouse experiment.

\section{References}

Abhilasha D, Joshi J (2009) Enhanced fitness due to higher fecundity, increased defence against a specialist and tolerance towards a generalist herbivore in an invasive annual plant. J Plant Ecol 2:77-86. https://doi.org/10.1093/jpe/rtp008

Ali JG, Agrawal AA (2012) Specialist versus generalist insect herbivores and plant defense. Trends Plant Sci 17:293-302. https://doi. org/10.1016/j.tplants.2012.02.006

Bates D, Machler M, Bolker BM, Walker SC (2015) Fitting Linear Mixed-Effects Models Using lme4. J Stat Softw 67:1-48

Bossdorf O, Schroder S, Prati D, Auge H (2004) Palatability and tolerance to simulated herbivory in native and introduced populations of Alliaria petiolata (Brassicaceae). Am J Bot 91:856-862. https ://doi.org/10.3732/ajb.91.6.856

Cabrera N, Sosa AJ, Julien M (2013) Phenrica littoralis (Bechyne, 1955) (Coleoptera: Chrysomelidae) a potential candidate for the biological control of alligator weed, Alternanthera philoxeroides (Martius) Grisebach (Amaranthaceae): redescription of the adult, first description of immature stages, and biological notes. Zootaxa 3686:543-555. https://doi.org/10.11646/zootaxa.3686.5.3

Cao L, Yang F, Tang S, Chen M (2014) Development of an artificial diet for three Lepidopteran insects. Chinese Journal of Applied Entomology 51:1376-1386. https://doi.org/10.7679/j. issn.2095-1353.2014.165 
Carmona D, Fornoni J (2013) Herbivores can select for mixed defensive strategies in plants. New Phytol 197:576-585. https://doi. org/10.1111/nph.12023

Cripps MG, Hinz HL, McKenney JL, Price WJ, Schwarzlaender M (2009) No evidence for an 'evolution of increased competitive ability' for the invasive Lepidium draba. Basic Appl Ecol 10:103112. https://doi.org/10.1016/j.baae.2008.03.001

De Mendiburu F (2009) Una herramienta de analisis estadistico para la investigacion agricola, Universidad Nacional de Ingenieria (UNI-PERU)

Dong BC, van Kleunen M, Yu FH (2018) Context-dependent parental effects on clonal offspring performance. Front Plant Sci 9:10. https ://doi.org/10.3389/fpls.2018.01824

Doorduin LJ, Vrieling K (2011) A review of the phytochemical support for the shifting defence hypothesis. Phytochem Rev 10:99-106. https://doi.org/10.1007/s11101-010-9195-8

Faizal A, Geelen D (2013) Saponins and their role in biological processes in plants. Phytochem Rev 12:877-893. https://doi. org/10.1007/s11101-013-9322-4

Fornoni J, Valverde PL, Nunez-Farfan J (2004) Population variation in the cost and benefit of tolerance and resistance against herbivory in Datura stramonium. Evolution 58:1696-1704

Fortuna TM, Eckert S, Harvey JA, Vet LEM, Muller C, Gols R (2014) Variation in plant defences among populations of a range-expanding plant: consequences for trophic interactions. New Phytol 204:989-999. https://doi.org/10.1111/nph.12983

Geng YP, van Klinken RD, Sosa A, Li B, Chen JK, Xu CY (2016) The relative importance of genetic diversity and phenotypic plasticity in determining invasion success of a clonal weed in the USA and China. Front Plant Sci 7:213. https://doi.org/10.3389/ fpls.2016.00213

Haukioja E, Koricheva J (2000) Tolerance to herbivory in woody vs. herbaceous plants. Evol Ecol 14:551-562. https://doi. org/10.1023/a:1011091606022

Huang W, Siemann E, Wheeler GS, Zou J, Carrillo J, Ding J (2010) Resource allocation to defence and growth are driven by different responses to generalist and specialist herbivory in an invasive plant. J Ecol 98:1157-1167. https://doi.org/10.111 $1 / j .1365-2745.2010 .01704 . x$

Hull-Sanders HM, Johnson RH, Owen HA, Meyer GA (2009) Influence of polyploidy on insect herbivores of native and invasive genotypes of Solidago gigantea (Asteraceae). Plant signaling \& behavior 4:893-895

Joshi J, Vrieling K (2005) The enemy release and EICA hypothesis revisited: incorporating the fundamental difference between specialist and generalist herbivores. Ecol Lett 8:704-714. https://doi. org/10.1111/j.1461-0248.2005.00769.x

Julien MH, Skarratt B, Maywald GF (1995) Potential geographicaldistribution of alligator weed and its biological-control by Agasicles hygrophila. J Aquat Plant Manag 33:55-60

Karban R, Baldwin IT (1997) Induced responses to herbivory. University of Chicago Press, Chicago

Keane RM, Crawley MJ (2002) Exotic plant invasions and the enemy release hypothesis. Trends Ecol Evol 17:164-170. https://doi. org/10.1016/s0169-5347(02)02499-0

Li HK, Wang R (1994) Biological control of alligator weed, Alternanthera philoxeroides, in central China by inoculative releases of Agasicles hygrophila [Col: Chrysomelidae] with artificial overwintering protection. Chin J Biol Control (In Chinese) 10:11-14

Lin TT, Klinkhamer PGL, Vrieling K (2015) Parallel evolution in an invasive plant: effect of herbivores on competitive ability and regrowth of Jacobaea vulgaris. Ecol Lett 18:668-676. https:// doi.org/10.1111/ele.12445

Liu M, Zhou F, Pan XY, Zhang ZJ, Traw MB, Li B (2018) Specificity of herbivore-induced responses in an invasive species,
Alternanthera philoxeroides (alligator weed). Ecol Evol 8:59-70. https://doi.org/10.1002/ece3.3615

Luginbill P (1928) The fall armyworm. United States Department of Agriculture Technical Bulletin of Botanical Research 34:1-91

Müller-Schärer H, Schaffner U, Steinger T (2004) Evolution in invasive plants: implications for biological control. Trends Ecol Evol 19:417-422. https://doi.org/10.1016/j.tree.2004.05.010

Maddox DM, Andres LA, Hennessey RD, Blackburn RD, Spencer NR (1971) Insects to control alligatorweed-invader of aquatic ecosystems in United-States. Bioscience 21:985-991. https://doi. org/10.2307/1296135

Oduor AMO, Lankau RA, Strauss SY, Gomez JM (2011) Introduced Brassica nigra populations exhibit greater growth and herbivore resistance but less tolerance than native populations in the native range. New Phytol 191:536-544. https://doi.org/10.111 1/j.1469-8137.2011.03685.X

Pan XY, Geng YP, Sosa A, Zhang WJ, Li B, Chen JK (2007) Invasive Alternanthera philoxeroides: Biology, ecology and management. Acta Phytotaxon Sin 45:884-900. https://doi.org/10.1360/aps06 134

Pan XY, Jia X, Chen J-K, Li B (2012) For or against: the importance of variation in growth rate for testing the EICA hypothesis. Biol Invasions 14:1-8. https://doi.org/10.1007/s10530-011-9941-x

Quintero C, Bowers MD (2013) Effects of insect herbivory on induced chemical defences and compensation during early plant development in Penstemon virgatus. Ann Bot 112:661-669. https://doi. org/10.1093/aob/mct011

R Development Core Team (2017) R: A language and environment for statistical computing. R Foundation for Statistical Computing, Vienna, Austria. Retrieved from https://www.R-project.org/

Rao GVR, Wightman JA, Rao DVR (1993) World review of the natural enemies and diseases of Spodoptera litura (f) (Lepidoptera, Noctuidae). Insect Science and Its Application 14:273-284

Ridenour WM, Vivanco JM, Feng Y, Horiuchi J-i, Callaway RM (2008) No evidence for trade-offs: Centaurea plants from America are better competitors and defenders. Ecol Monogr 78:369-386. https ://doi.org/10.1890/06-1926.1

Saldamando CI, Marquez EJ (2012) Approach to Spodoptera (Lepidoptera: Noctuidae) phylogeny based on the sequence of the cytocrhome oxydase I (COI) mitochondrial gene. Rev Biol Trop 60:1237-1248

Schwaegerle KE, Mclintyre H, Swingley C (2000) Quantitative genetics and the persistence of environmental effects in clonally propagated organisms. Evolution 54:452-461

Sosa A, Julien MH, Corda H (2004) New research on Alternanthera philoxeroides (alligator weed) in its South American native range. In: Cullen JM BD, Kriticos DJ, Lonsdale WM,Cullen JM, Briese DT, Kriticos DJ, Lonsdale WM (eds) Proceedings of the XI international symposium on biological control of weeds. CSIRO Entomology, Canberra

Strauss SY, Agrawal AA (1999) The ecology and evolution of plant tolerance to herbivory. Trends Ecol Evol 14:179-185. https://doi. org/10.1016/s0169-5347(98)01576-6

Strauss SY, Rudgers JA, Lau JA, Irwin RE (2002) Direct and ecological costs of resistance to herbivory. Trends Ecol Evol 17:278-285. https://doi.org/10.1016/s0169-5347(02)02483-7

van Klinken RD, Edwards OR (2002) Is host-specificity of weed biological control agents likely to evolve rapidly following establishment? Ecol Lett 5:590-596. https://doi.org/10.104 6/j.1461-0248.2002.00343.x

Wang GQ, Xu RS, Wang R (2011) Dynamic Relationships between structure and saponins accumulation in vegetative organs of Alternanthera philoxeroides Criseb. Bull Bot Res 31:686-691

Wang Y, Siemann E, Wheeler GS, Zhu L, Gu X, Ding J (2012) Genetic variation in anti-herbivore chemical defences in an 
invasive plant. J Ecol 100:894-904. https://doi.org/10.111 1/j.1365-2745.2012.01980.x

Willis AJ, Thomas MB, Lawton JH (1999) Is the increased vigour of invasive weeds explained by a trade-off between growth and herbivore resistance? Oecologia 120:632-640. https://doi.org/10.1007/ s004420050899

Zhang Z, Pan X, Blumenthal D, van Kleunen M, Liu M, Li B (2018) Contrasting effects of specialist and generalist herbivores on resistance evolution in invasive plants. Ecology 99:866-875. https ://doi.org/10.1002/ecy.2155
Zuur A, Ieno E, Walker N, Saveliev A, Smith G (2009) Mixed effects models and extensions in ecology with R. Springer, New York 